\title{
Saikosaponin D inhibits nasal inflammation by regulating T-box protein expressed in T cells, GATA-3, and ROR rt in a murine model of allergic rhinitis
}

\author{
chunhua piao $^{1}$, Thi Tho Bui ${ }^{2}$, Chang Ho Song ${ }^{1}$, and OK-Hee Chai ${ }^{1}$ \\ ${ }^{1}$ Jeonbuk National University Medical School \\ ${ }^{2}$ The University of Danang
}

May 5, 2020

\begin{abstract}
Aim: Saikosaponin D (SSD) is a commonly prescribed agent against inflammatory diseases in Asian countries. However, the anti-allergic inflammatory effect of SSD and its role in allergic rhinitis (AR) model is well known. In the present study, we investigated the anti-allergic and anti-inflammatory effects of SSD on the ovalbumin (OVA)-induced AR model. Methods: AR was induced in BALB/c mice by sensitization and treatment with OVA. Different concentrations of SSD and dexamethasone (Dex) were administered. The mice were then evaluated for the presence of nasal mucosal inflammation, the production of allergen-specific cytokine responses, as well as the histology of nasal mucosa. Results: Following SSD treatment, the nasal symptoms and the inflammation of nasal mucosa were significantly ameliorated. During AR, administering SSD to the AR mice appeared to balance the Type 1 helper T cells (Th1) to Th2 cytokine ratio by increasing and decreasing the percentage of Th1 and Th2 cytokines, respectively. Meanwhile, SSD also appeared to markedly inhibit Th17 cytokines and their related transcription factor, ROR?t, whereas the Th1 cytokines and their related transcription factor, T-bet, was significantly increased in NALF and lung homogenates in OVA-induced AR mice. Notably, SSD also reduced the levels of OVA-specific Immunoglobulin $\mathrm{E}(\mathrm{IgE})$ and IgG1, and increased the levels of IgG2a in serum. Conclusion: This study demonstrated that SSD has a significant anti-allergic inflammatory effect in the nasal cavity. SSD may represent an alternative therapeutic approach for the treatment of patients with AR through the regulation of T-bet, GATA-3, and ROR?t in inflammatory cells.
\end{abstract}

\section{What is already known}

- Anti-allergic and anti-histamine agents are commonly used in the treatment of allergic rhinitis.

- SSD has anti-inflammatory, immunomodulatory, antiviral, and anticancer activities.

- Drugs derived from natural products are known to induce fewer side effects than many synthetic drugs.

\section{What dose this study add}

- SSD improves AR clinical symptoms and regulates the Th1/Th2/Th17 cytokine and transcription factor imbalance.

- SSD exerts anti-allergic and anti-inflammatory activity via the $\mathrm{NF}-\varkappa \mathrm{B}$ pathway.

\section{What is the clinical significance}

Drugs derived from natural products are known to induce fewer side effects than many synthetic drugs. Our data may represent an alternative therapeutic approach for the treatment of patients with AR.

\section{Introduction}


Allergic rhinitis (AR) is an allergic inflammation of the nasal airways and is characterized by sneezing, rhinorrhea, itching, and nasal congestion. These clinical conditions are widely known to be induced by inflammatory mediators, including histamine, leukotrienes, and inflammatory cytokines, which are secreted by eosinophils (Pawankar, Mori, Ozu, \& Kimura, 2011). AR is one of the world's most common chronic illnesses among children and young adults, and its prevalence is estimated to range from approximately 10-50\% (Bellanti \& Wallerstedt, 2000). Anti-allergic and anti-histamine agents are commonly used in the treatment of allergic rhinitis; however, their effects are transient as they are only able to suppress the symptoms of the inflammatory response (Adamia, Jorjoliani, Khachapuridze, Katamadze, \& Chkuaseli, 2015). Therefore, it is important to develop novel therapeutic strategies that are effective for the treatment of AR (Mowen \& Glimcher, 2004). A T-box protein expressed in T cells (T-bet) is a transcription factor specifically expressed in Type 1 helper $\mathrm{T}$ (Th1) cells. GATA-3, on the other hand, has been shown to be an important factor in mediating allergic airway inflammation in vivo (Zhang et al., 1999). Effective therapeutic strategies would aim to either downregulate Th2 or Th17 cytokines or to downregulate the transcription factors that inhibit their production. Retinoic acid-related orphan nuclear receptor (ROR) $\gamma \mathrm{t}$ is a splice variant of ROR, which has been identified as an essential transcription factor during Th17 cellular differentiation (Chen et al., 2011). Patients with AR show an inflammatory Immunoglobulin E (IgE)-mediated response characterized by the Th2 immunological pattern together with mast cells, goblet cells, and eosinophil activation, as well as the release of inflammatory mediators, such as interleukin (IL)4 and IL-5, against allergen exposure (Durham et al., 1992). Th1/Th2 cells are associated with a series of immune and inflammatory diseases, such as bacterial and viral infectious diseases (Meagher, Wines, \& Cooper, 2002; Walker, Virchow, Bruijnzeel, \& Blaser, 1991). To date, the discovery of Th17 cells has introduced complexity into the existing Th1/Th2 balance paradigm and expanded our understanding of the pathogenesis of AR (S. B. Wang et al., 2014). Th17 cells are newly emerged immune/inflammatory cell subsets, which are now widely believed to be critical for the regulation of various chronic immune diseases (Halwani, Al-Muhsen, \& Hamid, 2013). Th17 cells enhance eosinophilic airway inflammation, which is mediated by Th2 cells (Wakashin et al., 2008). Nuclear factor-kappa B (NF- $\varkappa$ B) is a multicellular transcription factor, and it plays an important role in inflammatory and immune responses by regulating the gene expression of immune and inflammation-related cytokines and inflammatory mediators, which is a vital role in the initiation and perpetuation of allergic inflammation (Kumar, Takada, Boriek, \& Aggarwal, 2004; Wan \& Lenardo, 2010). The transcription factor T-bet drives Th1 differentiation, while the transcription factor GATA-3 drives Th2 differentiation. Natural products are regarded as abundant sources of novel drug candidates, and their pharmacological usefulness has been proven through decades of research (Newman \& Cragg, 2016). In addition, drugs derived from natural products are known to induce fewer side effects than many synthetic drugs (Mathur \& Hoskins, 2017). Saikosaponin D (SSD, chemical structure shown in Fig. 1A), one of the triterpenoid saponins derived from Bupleurum falcatum L, is a commonly prescribed agent against inflammatory diseases in China, Japan, and other Asian countries (Lu et al., 2012; Wong et al., 2013). Several studies have shown that SSD has anti-inflammatory, immunomodulatory, antiviral, and anticancer activities (H. W. Wang, Liu, Zhong, \& Fang, 2015). Other researchers have found that SSD exhibited an anti-proliferative effect on the activated T lymphocyte via suppression of the NF- $x \mathrm{~B}, \mathrm{NF}-\mathrm{AT}$, and AP-1 signaling pathways (Sun, Cai, Zhou, \& Xu, 2009; Wong et al., 2013). However, the mechanism underlying the anti-allergic effect of SSD on allergic rhinitis remains unclear. In the current study, we explore the protective effects and therapeutic efficacy of SSD on an ovalbumin (OVA)-induced AR mice model, which potentially involves the regulation of the Th1/Th2 and Th17 cellular responses.

\section{Methods}

\subsection{Animals}

Five-week-old male BALB/c mice were purchased from Damool Science (Dae-jeon, Korea). These mice were housed in a laminar air-conditioned room maintained at a temperature of $23 \pm 2$ and a relative humidity of $55+-10 \%$ with a 12 hour light/dark cycle. All animal experiments were performed in compliance with the guidelines on the care and use of laboratory animals and were approved by the Animal Research Committee of Jeonbuk National University (CBN2016-37). 


\subsection{Induction of AR mice model and treatment}

Mice were randomly divided into four groups: the control group, OVA group, $10 \mathrm{mg} / \mathrm{kg}$ of SSD, and Dexamethasone (Dex, $2.5 \mathrm{mg} / \mathrm{kg}$ ) group. The schedule for the OVA-induced AR animal model and treatment is summarized in Figure 1B. Briefly, mice were sensitized by intraperitoneal injection of $50 \mu \mathrm{g}$ of OVA (Grade V, Sigma, St. Louis, MO, USA) emulsified in $1 \mathrm{mg}$ of aluminum hydroxide (Thermo Scientific, Rockford, MD, USA) in a total volume of $200 \mu \mathrm{l}$ on days 1,8 , and 15 . After one week of the last sensitization (days 22 to 26$)$, mice were treated with an intranasal instillation of OVA $(10 \mathrm{mg} / \mathrm{ml}, 20 \mu \mathrm{l} / \mathrm{nostril})$. From day 16, mice received SSD (Sigma, St. Louis, MO, USA, $10 \mathrm{mg} / \mathrm{kg}$ body weight) and Dex (Innovative Research of America, Toledo, Ohio, USA) dissolved in saline in a total volume of $200 \mu \mathrm{l}$ and administered by oral gavage 1 hour before every OVA treatment, every day for 11 consecutive days. Mice of the control group were treated with saline but without sensitization and OVA treatment. Mice were sacrificed $24 \mathrm{~h}$ after the last OVA treatment on day 27 to investigate the anti-inflammatory effect of SSD on OVA-induced AR mice.

\subsection{Analysis of nasal lavage fluid (NALF)}

Twenty-four hours after the last OVA challenge, the mice were sacrificed. The trachea was partially resected, a catheter was inserted from the trachea into the nasopharynx, and the nasal cavity was gently perfused with $1 \mathrm{ml}$ of saline from the choana to the nostril. The NALF was centrifuged at 10,000 $\times \mathrm{g}$ for $10 \mathrm{~min}$, and the supernatants were stored at -80 . The total NALF cells were counted using the double-blind counting system with a hemocytometer using trypan blue staining. Differential cell counts were determined with cytospin cell preparations, which were made by transferring NALF onto clean glass slides using a cytospin device (Centrifuge 5403, Eppendorf, Hamburg, Germany) for 10 min and staining with Diff-Quik (Sysmex Co., Kobe, Japan), according to the manufacturer's instructions.

\subsection{Evaluation of nasal symptoms}

Nasal symptoms were evaluated by counting the frequencies of nasal rubbing and sneezing that occurred in the 20-minute observation period and then counted by blinded observers to evaluate early allergic responses.

\subsection{Histological examination}

After performing the NALF, the heads of the mice were removed and then fixed in $10 \%$ neutral buffered formalin for 3 days and then decalcified in an ethylenediamine triacetic acid decalcifying solution for 5 days. The specimens were dehydrated with ethyl alcohol and xylene and embedded in paraffin, then embedded in paraffin wax and sectioned at 5 - $\mu \mathrm{m}$ thickness. The lung lobes were removed for histological examination and were fixed in $10 \%$ paraformaldehyde for 3 days. The nasal and lung tissues were coronally sectioned into 5 - $\mu \mathrm{m}$ slices for histological assessment. Subsequently, the number of inflammatory cells was counted, and the epithelial damage was evaluated on randomly selected high-power fields at $400 \times$ magnification.

Hematoxylin and eosin (HE, Sigma Aldrich, St. Louis, MO, USA) staining was used to assess the general morphological structure of the nasal and lung tissues.

Periodic acid-Schiff (PAS, Sigma, St. Louis, MO) staining was used for goblet cell hyperplasia.

Giemsa staining (Sigma, St. Louis, MO) was used for mast cell and eosinophil infiltration in nasal mucosa. Eosinophils and mast cells were counted in the nasal septum and lateral process.

Masson's trichrome staining was used to reveal the sub-epithelial deposition of collagen in the lung tissue.

\subsection{Measurement of Th1/Th2/Th17 cytokines and related transcription factor levels}

In the Th1 response, the levels of anti-inflammatory cytokines (Th1-associated cytokine), such as IFN- $\gamma$ and IL-12 levels, and the relative transcription factor T-bet in NALF and lung homogenates were assayed using enzyme-linked immunosorbent assay kits (ELISA, R\&D Systems, Minneapolis, USA), following the manufacturer's instructions. 
In the Th2 response, we examined the secretion of the inflammatory cytokines (Th2-associated cytokine) TNF- $\alpha$, IL-4, and IL-5 and relative transcription factor GATA-3 in NALF and lung homogenates were measured using ELISA (R\&D Systems, Minneapolis, USA), following manufacturer's instructions.

In the Th17 response, Th17-associated cytokines, IL-17, and relative transcription factor ROR $\gamma$ t in NALF and lung homogenates were measured using ELISA (R\&D Systems, Minneapolis, USA), following manufacturer's instructions.

\subsection{Measurement of OVA-specific immunoglobulins}

The blood specimens were collected from the retro-orbital plexus and centrifuged at 1,000 x $\mathrm{g}$ for $10 \mathrm{~min}$ at 4 (Centrifuge 5403; Eppendorf, Hamburg, Germany) to obtain the serum. Then, the concentrations of OVA-specific IgE, OVA-specific IgG1, and OVA-specific IgG2 $\alpha$ levels were measured in each group using ELISA (R\&D Systems, Minneapolis, USA), following manufacturer's instructions.

\subsection{Measurement of the NF-kB signaling pathway}

The lung homogenates were centrifuged at $1,000 \times \mathrm{g}$ for $15 \mathrm{~min}$ at $4{ }^{\circ} \mathrm{C}$. The supernatants of NF-KB p65 and P- NF- $\mathrm{KB}$, both in NALF and lung homogenates, were obtained for analysis.

\subsection{Statistical analysis}

The results were analyzed using Graph Pad Prism software (v5.0, La Jolla, CA, USA). The data were presented as mean \pm SEMs. The statistical significance of the differences among the groups was performed using one-way ANOVA, followed by the Tukey's test. Statistical significance was defined at the $95 \%$ confidence level $(P<0.05)$.

\subsection{Materials}

OVA (grade VI), and SSD were purchased from Sigma-Aldrich (Sigma, St. Louis, MO, USA). Aluminum hydroxide adjuvant was purchased from Pierce (Thermo Scientific, Rockford, MD, USA) and Dex was purchased from Innovative Research of America (Innovative Research of America, Toledo, Ohio, USA).

\section{Results}

\subsection{Inhibitory effects of SSD on clinical symptoms of OVA-induced AR mice}

In animal models, early allergic responses have been quantitated by the nasal rubbing and sneezing score. To examine the regulatory effect of SSD in the OVA-induced AR mice model, after $400 \mu \mathrm{g}$ of OVA intranasal treatment, we measured the frequency of nasal sneezing and the rubbing behaviors were recorded during a 20minute period by blinded observers on days 22 to 26 . The OVA-induced AR mice showed a significant increase in sneezing and nasal rubbing as compared to the control mice (Fig. 1C). However, oral administration of 10 $\mathrm{mg} / \mathrm{kg} /$ day SSD revealed a tendency to decrease the sneezing and nasal rubbing (Fig. 1C). SSD treatments considerably improved nasal symptoms compared to those of OVA-induced AR mice. These results suggest that treatment with SSD may ameliorate the symptoms associated with AR.

3.2 Inhibitory effects of SSD on the infiltration of inflammatory cells in the NALF of OVAinduced AR mice

To evaluate the underlying mechanism of SSD on the level of inflammatory cells in OVA-induced AR mice, the total number of cells and the differential inflammatory cells in NALF were counted. OVA-induced AR mice exhibited a more significant increase in total cells and inflammatory cells of NALF than mice of the control group (Fig. 1D, E). In contrast, oral administration of $10 \mathrm{mg} / \mathrm{kg} /$ day SSD and $2.5 \mathrm{mg} / \mathrm{kg} /$ day Dex subsequently reduced the total cell number and the infiltration of eosinophils, epithelial cells, and other inflammatory cells in NALF (Fig. 1D, E).

3.3 Inhibitory effects of SSD on the histopathological changes of nasal mucosal tissues and lungs of OVA-induced AR mice 
To evaluate the effects of SSD administration on the infiltration of inflammatory cells in nasal mucosal tissues and lung tissues, we next examined the histological changes of OVA-induced AR mice with HE, PAS, Giemsa, and Masson's trichrome. As shown in Fig. 2A, OVA treatment caused remarkable histological alterations, such as epithelial disruption and edema. Infiltration of eosinophils and mast cells, as well as goblet cell hyperplasia, was significantly increased compared to the control mice. (Fig. 2A). The mucosal thickness of the nasal septum was significantly higher in OVA-induced AR mice than in control mice. However, SSD treatment notably mitigated epithelial disruption and inhibited the infiltration of eosinophils, mast cells, and the hyperplasia of goblet cells.

The histological changes in lung tissues in OVA-induced AR mice were then evaluated, and the level of general pathological alterations was examined. Histological observations revealed that there was a more severe infiltration of inflammatory cells around the respiratory tract and blood vessels than in the control group. While SSD treatment showed a significantly reduced infiltration (Fig. 2B). These results suggest that SSD may alleviate allergic responses in OVA-treated mice by attenuating the inflammatory cells infiltration both in the upper and lower airway.

3.4 Inhibitory effects of SSD on the serum levels of OVA-specific IgE, IgG1, and IgG2a of OVA-induced AR mice

In order to investigate the therapeutic impact of SSD on OVA-specific immune responses, we determined the serum levels of OVA-specific immunoglobulin in OVA-induced AR mice. As shown in Fig. 3, both levels of OVA-specific IgE and IgG1 in OVA-induced AR mice were markedly increased. However, treatment with SSD significantly inhibited OVA-specific IgE and IgG1 production (Fig. 3). Moreover, the level of OVAspecific IgG2a was slightly up-regulated by SSD treatment compared with OVA-induced AR mice. These results indicate that SSD might have anti-allergic activity via suppression of allergic mediator production.

3.5 Effects of SSD on the levels of Th1, Th2, and Th17 cytokines in NALF and lung homogenates of OVA-induced AR mice

To further evaluate the effect of SSD on anti-allergic responses in the OVA-induced AR mice model, the Th1/Th2/Th17 levels of secreted cytokines in NALF and lung homogenates were assessed. First, we examined the secretion of the Th2-associated cytokines, including TNF- $\alpha$, IL-4, and IL-5, using ELISA. OVAinduced AR mice showed increased levels of TNF- $\alpha$, IL-4, and IL-5 in NALF compared to control mice; however, SSD treatment significantly decreased the cytokine levels (Fig 4A, B). Moreover, the anti-inflammatory effect of Th1-associated cytokines, such as IFN- $\gamma$ and IL-12, were measured using ELISA. The OVA-induced release of IFN- $\gamma$ was significantly increased by SSD and Dex treatment (Fig 4A, B), while the production of IL-12 did not show any significant difference (Fig. 4A, B). Th17-associated cytokine IL-17 was increased in the AR mice (Fig. 4A, B). In contrast, SSD treatment significantly diminished IL-17 production in the NALF and Dex groups (Fig. 4A, B).

Since the administration of SSD suppressed the levels of IL-17 in NALF, we investigated the possibility that Th17 production was down-regulated in the lung tissue. As expected, OVA-induced AR mice dramatically increased IL-17 levels in lung homogenates compared to the control group. However, the administration of SSD markedly attenuated the production of IL-17 (Fig. 4A, B). These results were consistent with the reduced infiltration of inflammatory cells in NALF, and nasal tissues of SSD treated mice via the modulation of the Th1/Th2/Th17 cytokines.

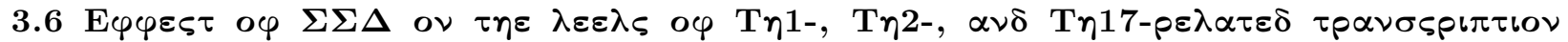

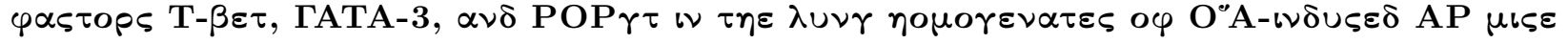

It was hypothesized that the mechanisms underlying the anti-allergic effects of SSD involve the regulation of transcription factors, including T-bet, GATA-3, and ROR $r$ t, during Th1, Th2, and Th17 cellular differentiation. The expression of the specific transcription factors, T-bet, GATA-3, and ROR rt in the nucleus, was used to identify whether Th1, Th2, Th17 cells, respectively, were altered in the lung tissue following SSD and Dex administration. The production of GATA-3 in the OVA-induced AR mice increased significantly 
(Fig. 4C). In contrast, the T-bet expression levels in the OVA-induced AR mice were lower than those in the SSD and Dex groups. The expression of GATA-3 expression was decreased in the SSD group compared to the control group. There were no significant differences in the expression levels of T-bet between the OVA and SSD groups. Similar effects were observed in the Th17 transcription factor ROR $\gamma$ t. The expression of ROR rt appeared to be up-regulated in OVA-induced AR mice. Conversely, treatment with SSD and Dex ameliorated this effect.

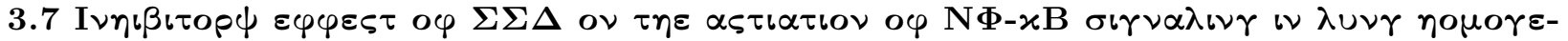

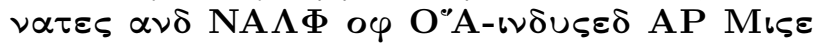

The signaling pathway of NF- $\chi \mathrm{B}$, a transcription factor, is important in the synthesis and release of inflammatory mediators by activated mast cells during allergic inflammation (Cho et al., 2014; Jung, Jung, Kim, Kang, \& Park, 2012). Therefore, to assess the effect of SSD on the signaling pathway, NF- $x \mathrm{~B}$ and P-NF- $\varkappa \mathrm{B}$, both in NALF and lung homogenates were analyzed by ELISA. The results showed that in OVA-induced AR mice, the levels of NF- $\chi \mathrm{B}$ in NALF and lung homogenates were significantly increased as compared to control mice; however, their levels were significantly decreased by SSD treatment (Fig. 4D). In addition, as a marker of NF- $\varkappa \mathrm{B}$ activation, degradation levels of P-NF- $\chi \mathrm{B}$ in NALF and lung homogenates were examined. OVA-induced AR mice showed an increased level of P-NF- $\chi \mathrm{B}$ degradation in NALF and lung homogenates as compared to control mice. Moreover, we found that SSD treatment down-regulated the levels of P-NF- $x \mathrm{~B}$ in NALF and lung homogenates as compared to OVA-induced AR. These findings indicate the potential role of the NF- $x$ B pathway in the suppression of inflammatory mediators by SSD in OVA-induced AR.

\section{Discussion}

The clinical symptoms of AR include sneezing, rubbing, rhinorrhea, lacrimation, and nasal congestion, which mainly originate from mast cells and other inflammatory cells (Mandhane, Shah, \& Thennati, 2011). These symptoms are presumed to be triggered by various mediators such as cytokines (IL-4, TNF- $\alpha$ ), prostaglandins, NO from mast cells, and other inflammatory cells (Fu, Dai, Wang, \& Zhang, 2003). In the present study, we investigated the effects of SSD treatment on the nasal mucosa in an AR mouse model. We demonstrated that SSD treatment was associated with significantly decreased clinical symptoms as well as an increase in the number of disrupted epithelial cells in the NALF, serum OVA-specific immunoglobulin levels, Th2, and Th17 cytokines levels. Moreover, SSD treatment inhibited the infiltration of eosinophils, mast cells, goblet cells, and epithelial cells into the nasal mucosa. In addition, goblet cell hyperplasia and collagen deposition were also alleviated in lung tissue. The down-regulation of transcription factor GATA-3, ROR $\gamma \mathrm{t}$ expression, and the up-regulation of T-bet expression were observed in the SSD group. We further demonstrated that SSD was able to suppress the release of allergic mediators by blocking the NF- $x \mathrm{~B}$ signaling pathway.

Under the physiological conditions of an allergic response, Th1 and Th2 populations exist in equilibrium and cross-regulate each other, and the Th1/Th2 balance is reported to be necessary for the maintenance of immune homeostasis (Gotoh, 2015; Ma et al., 2013). GATA-3 is a transcription factor that is specifically expressed in Th2 cells, whereas T-bet is a transcription factor specifically expressed in Th1 cells. GATA3 and T-bet have been reported to modulate gene expression during $\mathrm{T}$ cell differentiation, thus serving a critical role in the development of Th1 and Th2 lineages (Han et al., 2013; Ku et al., 2015). The present study revealed that in OVA-induced AR mice, GATA-3 expression was markedly up-regulated in lung tissue compared to control mice. Notably, GATA-3 expression levels were markedly decreased following treatment with SSD. Conversely, T-bet levels were up-regulated following SSD administration; however, there was no significant difference compared to OVA-induced AR mice. The present findings demonstrate that the expression of GATA-3 and T-bet may be altered during allergic immune responses and may be implicated in the regulation of Th1/Th2 differentiation. Following the retroviral vector-mediated transduction of ROR $\gamma \mathrm{t}$ into naive T cells, Th17 cell development was enhanced, therefore suggesting that ROR $r$ t may be essential for Th17 cellular proliferation (Korn, Bettelli, Oukka, \& Kuchroo, 2009). The present study revealed that the protein expression of ROR $r$ t was down-regulated following SSD administration.

AR is one of the most common chronic conditions in the adult and pediatric population, affecting 10-30\% 
of adults and 20-40\% of children (Meltzer et al., 2009). Therefore, the development of new and promising therapeutic options for the treatment of AR is required. In the present study, we demonstrated that OVA treatment of AR mice induced nasal airway remodeling compared to control mice via increased epithelial disruption, mucosa edema, eosinophil and mast cell infiltration, goblet cell hyperplasia in the nasal mucosa, OVA-specific immunoglobulins, Th2, and Th17 polarization, and NF- $x$ B signaling pathway activation in nasal epithelial cells. In the present study, we assessed the anti-allergic and anti-inflammatory activities of SSD in the OVA-induced AR mice to elucidate the effects of SSD on inflammation-related NF- $x$ B pathways. Here, we demonstrate for the first time, that SSD, which is a commonly prescribed agent against inflammatory diseases, significantly attenuated allergic responses.

SSD is considered one of the major active components isolated and identified from the herb (Xu et al., 2012). Protection against CCl4-induced inflammation and fibro-genesis by SSD was correlated with the down-regulation of pro-inflammatory cytokines, such as TNF- $\alpha$, IL-1 $\beta$, and IL-6, and the up-regulation of the anti-inflammatory cytokine, IL-10 (Wu, Tam, Tsai, Chang, \& Chao, 2010). A previous report suggested that IL-17 has the potential to promote airway inflammatory cell infiltration (Molet et al., 2001). The pathogenesis of AR is characterized by the secretion of many cytokines and immune cells. IL-4 expression is related to the differentiation of Th2 lymphocytes, the synthesis of $\operatorname{IgE}$, and the hypersecretion of mucus (Pawankar et al., 2011). IL-5 plays a role in eosinophilic inflammation (Foster, Hogan, Ramsay, Matthaei, \& Young, 1996). In this study, we demonstrated that SSD diminished Th2 cytokine levels, including TNF- $\alpha$, IL-4, and IL-5, as well as Th17 cytokine IL-17 expression levels in NALF and lung homogenates by inhibiting $\mathrm{NF}-x \mathrm{~B}$ activation.

$\mathrm{NF}-\chi \mathrm{B}$ regulates the transcription of pro-inflammatory cytokines (Hoffmann \& Baltimore, 2006). I $\chi \mathrm{B}$ is rapidly removed by $\mathrm{IKK} \alpha / \beta$ and activated $\mathrm{NF}-\varkappa \mathrm{B}$ is released and then translocated to the nucleus, where it activates the transcription of target genes (Hayden \& Ghosh, 2004). The effect of SSD treatment supported the hypothesis that the expression of these pro-inflammatory cytokines is $\mathrm{NF}-\chi \mathrm{B}$ dependent. These results revealed that SSD suppressed NF- $\varkappa \mathrm{B}$ and $\mathrm{P}-\mathrm{NF}-\varkappa \mathrm{B}$ activation. In addition, the NF- $\varkappa \mathrm{B}$ dependent increase in the levels of inflammatory cytokines was markedly inhibited. Therefore, we postulate that SSD has an anti-inflammatory effect, which acts through the inhibition of NF- $x \mathrm{~B}$ activation.

The OVA sensitization and treatment of mice led to an increase in the levels of antigen-specific immunoglobulins in serum and the infiltration of inflammatory cells in the epithelium and sub-epithelium of the nasal mucosa (Bahekar, Shah, Ayer, Mandhane, \& Thennati, 2008). The released IgE is responsible for increased production of leukotrienes and prostaglandins, which in turn attract eosinophils, increase microvascular leakage, edema, and mucous secretion (Smith, 1999). Our study revealed that SSD remarkably inhibited OVA-specific IgE and IgG1 levels and increased the production of IgG2a in OVA-induced mice. These results demonstrated that SSD displays an anti-allergic activity against allergic responses.

Mast cells and eosinophils regulate local immune and inflammatory responses, and their accumulation in blood and tissue is associated with several inflammatory diseases (Tian et al., 2017). The recruitment of eosinophils in AR is reportedly initiated by Th2-type cytokines (Juneja \& Parmar, 2013). Our results demonstrated that SSD inhibited the infiltration of mast cells, eosinophils, and goblet cell hyperplasia into the nasal tissues of OVA-induced AR mice. Therefore, we postulate that SSD undergoes anti-allergic and anti-inflammatory reactions through the inhibition of inflammatory cell infiltration, of both the nasal and lung tissues.

In summary, our findings provide in vivo evidence that the anti-allergic and anti-inflammatory agent SSD significantly diminishes allergic reactions by alleviating the symptoms of OVA-induced AR, by mediating the Th1/Th2 cell balance, via regulation of the T-bet, GATA-3, and ROR $r$ t expression levels. Therefore, SSD may represent an alternative therapeutic approach for the treatment of patients with AR.

\section{Conflicts of Interest}

None. 


\section{Data availability statement}

The data used to support the findings of this study are available from the corresponding author upon request.

\section{Author contributions}

OHC designed the experiments. CHP and TTB performed the experiments. CHP and CHS analyzed the data and performed the biological analysis. The manuscript was written by CHP and OHC. All authors discussed the results and reviewed the manuscript.

\section{Reference}

Adamia, N., Jorjoliani, L., Khachapuridze, D., Katamadze, N., \& Chkuaseli, N. (2015). Allergic Diseases and Asthma in Adolescents.Georgian Med News(243), 58-62. Retrieved fromhttps://www.ncbi.nlm.nih.gov/pubmed/26087732Bahekar, P. C., Shah, J. H., Ayer, U. B., Mandhane, S. N., \& Thennati, R. (2008). Validation of guinea pig model of allergic rhinitis by oral and topical drugs. Int Immunopharmacol, 8(11), 1540-1551. doi:10.1016/j.intimp.2008.06.010 Bellanti, J. A., \& Wallerstedt, D. B. (2000). Allergic rhinitis update: Epidemiology and natural history. Allergy Asthma Proc, 21(6), 367-370. Retrieved fromhttps://www.ncbi.nlm.nih.gov/pubmed/11191103Chen, Z. J., Lin, F., Gao, Y. Y., Li, Z. Y., Zhang, J., Xing, Y., . . . Li, B. (2011). FOXP3 and ROR gamma t: Transcriptional regulation of Treg and Th17. International Immunopharmacology, 11(5), 536542. doi:10.1016/j.intimp.2010.11.008 Cho, M. S., Park, W. S., Jung, W. K., Qian, Z. J., Lee, D. S., Choi, J. S., . . . Choi, I. W. (2014). Caffeic acid phenethyl ester promotes anti-inflammatory effects by inhibiting MAPK and NF-kappaB signaling in activated HMC-1 human mast cells. Pharm Biol, 52(7), 926-932. doi:10.3109/13880209.2013.865243 Durham, S. R., Ying, S., Varney, V. A., Jacobson, M. R., Sudderick, R. M., Mackay, I. S., . . . Hamid, Q. A. (1992). Cytokine messenger RNA expression for IL-3, IL-4, IL5 , and granulocyte/macrophage-colony-stimulating factor in the nasal mucosa after local allergen provocation: relationship to tissue eosinophilia.J Immunol, 148(8), 2390-2394. Retrieved fromhttps://www.ncbi.nlm.nih.gov/pubmed/1560199Foster, P. S., Hogan, S. P., Ramsay, A. J., Matthaei, K. I., \& Young, I. G. (1996). Interleukin 5 deficiency abolishes eosinophilia, airways hyperreactivity, and lung damage in a mouse asthma model. J Exp Med, 183(1), 195-201. Retrieved fromhttps://www.ncbi.nlm.nih.gov/pubmed/8551223Fu, L. J., Dai, Y., Wang, Z. T., \& Zhang, M. (2003). Inhibition of experimental allergic rhinitis by the n-butanol fraction from the anomalous fruits of Gleditsia sinensis. Biol Pharm Bull, 26(7), 974-977. Retrieved fromhttps://www.ncbi.nlm.nih.gov/pubmed/12843621 Gotoh, M. (2015). [Allergen Immunotherapy for Allergic Rhinitis].Arerugi, 64(5), 693-698; quiz 698-699. Retrieved fromhttps://www.ncbi.nlm.nih.gov/pubmed/26108750Halwani, R., Al-Muhsen, S., \& Hamid, Q. (2013). T helper 17 cells in airway diseases: from laboratory bench to bedside. Chest, 143(2), 494-501. doi:10.1378/chest.12-0598 Han, L. N., Guo, S. L., Li, T. L., Ding, G. L., Zhang, Y. J., \& Ma, J. L. (2013). Effect of immune modulation therapy on cardiac function and T-bet/GATA-3 gene expression in aging male patients with chronic cardiac insufficiency. Immunotherapy, 5(2), 143-153. doi:10.2217/imt.12.139 Hayden, M. S., \& Ghosh, S. (2004). Signaling to NF-kappaB. Genes Dev, 18(18), 2195-2224. doi:10.1101/gad.1228704 Hoffmann, A., \& Baltimore, D. (2006). Circuitry of nuclear factor kappaB signaling. Immunol Rev, 210, 171-186. doi:10.1111/j.01052896.2006.00375.x Juneja, L., \& Parmar, H. S. (2013). Ovalbumin induced allergic rhinitis and development of prediabetes to rats: possible role of Th2 cytokines.Inflamm Allergy Drug Targets, 12(3), 199-205. Retrieved fromhttps://www.ncbi.nlm.nih.gov/pubmed/23477548Jung, H. W., Jung, J. K., Kim, Y. H., Kang, J. S., \& Park, Y. K. (2012). Effect of KOB03, a polyherbal medicine, on ovalbumin-induced allergic rhinitis in guinea pigs. Chin Med, $7(1), 27$. doi:10.1186/1749-8546-7-27 Korn, T., Bettelli, E., Oukka, M., \& Kuchroo, V. K. (2009). IL-17 and Th17 Cells. Annu Rev Immunol, 27, 485-517. doi:10.1146/annurev.immunol.021908.132710 Ku, C. J., Lim, K. C., Kalantry, S., Maillard, I., Engel, J. D., \& Hosoya, T. (2015). A 
monoallelic-to-biallelic T-cell transcriptional switch regulates GATA3 abundance. Genes Dev, 29(18), 1930-1941. doi:10.1101/gad.265025.115 Kumar, A., Takada, Y., Boriek, A. M., \& Aggarwal, B. B. (2004). Nuclear factor-kappa B: its role in health and disease. Journal of Molecular Medicine-Jmm, 82(7), 434-448. doi:10.1007/s00109-004-0555-y Lu, C. N., Yuan, Z. G., Zhang, X. L., Yan, R., Zhao, Y. Q., Liao, M., \& Chen, J. X. (2012). Saikosaponin a and its epimer saikosaponin d exhibit anti-inflammatory activity by suppressing activation of NF-kappaB signaling pathway. Int Immunopharmacol, 14 (1), 121-126. doi:10.1016/j.intimp.2012.06.010 Ma, C., Ma, Z., Liao, X. L., Liu, J., Fu, Q., \& Ma, S. (2013). Immunoregulatory effects of glycyrrhizic acid exerts anti-asthmatic effects via modulation of Th1/Th2 cytokines and enhancement of CD4(+)CD25(+)Foxp3+ regulatory T cells in ovalbumin-sensitized mice.J Ethnopharmacol, 148(3), 755-762. doi:10.1016/j.jep.2013.04.021 Mandhane, S. N., Shah, J. H., \& Thennati, R. (2011). Allergic rhinitis: an update on disease, present treatments and future prospects. Int Immunopharmacol, 11(11), 1646-1662. doi:10.1016/j.intimp.2011.07.005 Mathur, S., \& Hoskins, C. (2017). Drug development: Lessons from nature. Biomed Rep, 6(6), 612-614. doi:10.3892/br.2017.909 Meagher, L. J., Wines, N. Y., \& Cooper, A. J. (2002). Atopic dermatitis: review of immunopathogenesis and advances in immunosuppressive therapy. Australas J Dermatol, 43(4), 247-254. Retrieved fromhttps://www.ncbi.nlm.nih.gov/pubmed/12423430Meltzer, E. O., Blaiss, M. S., Derebery, M. J., Mahr, T. A., Gordon, B. R., Sheth, K. K., . . . Boyle, J. M. (2009). Burden of allergic rhinitis: results from the Pediatric Allergies in America survey. $J$ Allergy Clin Immunol, 124(3 Suppl), S43-70. doi:10.1016/j.jaci.2009.05.013 Molet, S., Hamid, Q., Davoine, F., Nutku, E., Taha, R., Page, N., . . . Chakir, J. (2001). IL-17 is increased in asthmatic airways and induces human bronchial fibroblasts to produce cytokines. J Allergy Clin Immunol, 108 (3), 430-438. doi:10.1067/mai.2001.117929 Mowen, K. A., \& Glimcher, L. H. (2004). Signaling pathways in Th2 development. Immunol Rev, 202, 203-222. doi:10.1111/j.01052896.2004.00209.x Newman, D. J., \& Cragg, G. M. (2016). Natural Products as Sources of New Drugs from 1981 to 2014. J Nat Prod, 79(3), 629-661. doi:10.1021/acs.jnatprod.5b01055 Pawankar, R., Mori, S., Ozu, C., \& Kimura, S. (2011). Overview on the pathomechanisms of allergic rhinitis. Asia Pac Allergy, 1(3), 157-167. doi:10.5415/apallergy.2011.1.3.157 Smith, L. J. (1999). Pharmacology and safety of the leukotriene antagonists. Clin Rev Allergy Immunol, 17(1-2), 195-212. doi:10.1007/BF02737604 Sun, Y., Cai, T. T., Zhou, X. B., \& Xu, Q. (2009). Saikosaponin a inhibits the proliferation and activation of $\mathrm{T}$ cells through cell cycle arrest and induction of apoptosis. Int Immunopharmacol, 9(7-8), 978-983. doi:10.1016/j.intimp.2009.04.006 Tian, B. P., Xia, L. X., Bao, Z. Q., Zhang, H., Xu, Z. W., Mao, Y. Y., . . . Shen, H. H. (2017). Bcl-2 inhibitors reduce steroid-insensitive airway inflammation. J Allergy Clin Immunol, 140(2), 418-430. doi:10.1016/j.jaci.2016.11.027 Wakashin, H., Hirose, K., Maezawa, Y., Kagami, S., Suto, A., Watanabe, N., . . . Nakajima, H. (2008). IL-23 and Th17 cells enhance Th2-cell-mediated eosinophilic airway inflammation in mice. Am J Respir Crit Care Med, 178(10), 1023-1032. doi:10.1164/rccm.200801-086OC Walker, C., Virchow, J. C., Jr., Bruijnzeel, P. L., \& Blaser, K. (1991). T cells and asthma. II. Regulation of the eosinophilia of asthma by $\mathrm{T}$ cell cytokines. Int Arch Allergy Appl Immunol, 94(1-4), 248-250. Retrieved fromhttps://www.ncbi.nlm.nih.gov/pubmed/1937884Wan, F., \& Lenardo, M. J. (2010). The nuclear signaling of NF-kappaB: current knowledge, new insights, and future perspectives. Cell Res, 20(1), 24-33. doi:10.1038/cr.2009.137 Wang, H. W., Liu, M., Zhong, T. D., \& Fang, X. M. (2015). Saikosaponin-d attenuates ventilator-induced lung injury in rats. Int J Clin Exp Med, 8(9), 15137-15145. Retrieved fromhttps://www.ncbi.nlm.nih.gov/pubmed/26628997Wang, S. B., Deng, Y. Q., Ren, J., Xiao, B. K., Liu, Z., \& Tao, Z. Z. (2014). Exogenous interleukin-10 alleviates allergic inflammation but inhibits local interleukin-10 expression in a mouse allergic rhinitis model. BMC Immunol, 15, 9. doi:10.1186/1471-2172-15-9 Wong, V. K., Zhang, M. M., Zhou, H., Lam, K. Y., Chan, P. L., Law, C. K., . . . Liu, L. (2013). Saikosaponin-d Enhances the Anticancer Potency of TNF-alpha via Overcoming Its Undesirable Response of Activating NF-Kappa B Signalling in Cancer Cells. Evid Based Complement Alternat Med, 2013, 745295. 
doi:10.1155/2013/745295 Wu, S. J., Tam, K. W., Tsai, Y. H., Chang, C. C., \& Chao, J. C. (2010). Curcumin and saikosaponin a inhibit chemical-induced liver inflammation and fibrosis in rats. Am J Chin Med, 38(1), 99-111. doi:10.1142/S0192415X10007695 Xu, L., Song, R., Tian, J. X., Tian, Y., Liu, G. Q., \& Zhang, Z. J. (2012). Analysis of saikosaponins in rat plasma by anionic adducts-based liquid chromatography tandem mass spectrometry method. Biomed Chromatogr, 26 (7), 808-815. doi:10.1002/bmc.1734 Zhang, D. H., Yang, L. Y., Cohn, L., Parkyn, L., Homer, R., Ray, P., \& Ray, A. (1999). Inhibition of allergic inflammation in a murine model of asthma by expression of a dominant-negative mutant of GATA-3.Immunity, 11(4), 473-482. doi:Doi 10.1016/S1074-7613(00)80122-3

Figure Legends:

Figure 1. Experimental protocol for AR, nasal symptoms, and inflammation of OVA-induced AR mice. (A) Chemical structure of saikosaponin D (SSD). (B) Experimental protocol. (C) The frequencies of total rubbing and sneezing numbers for 5 days were evaluated by counting that occurred in the 20-minute period after OVA treatment without anesthesia. (C) Total and differential cell numbers were counted using a hemocytometer. (D) Cytospin cell preparations were made by NALF and stained with Diff-Quik. Scale bar $=100 \mu \mathrm{m}$. $\# P<0.05$, \#\# $P<0.01$, \#\#\# $P<0.001$, significant differences of the control group. Values are presented as the mean $\pm \mathrm{SD}\left(\mathrm{n}=6\right.$ per group). ${ }^{*} \mathrm{P}<0.05,{ }^{* *} \mathrm{P}<0.01,{ }^{* * *} \mathrm{P}<0.001$, significant differences of the OVA group.

Figure 2. Effect of SSA on histological changes in the nasal and lung tissues of OVA-induced AR mice. (A) The general histology and epithelium thickness of the nasal mucosa, HE staining; Infiltration of the eosinophils, Sirius Red staining; Goblet cells hyperplasia, PAS staining; Mast cells immigration to the epithelium, Giemsa staining; Lung tissues from each group were stained with HE. (B) The general histology and epithelium thickness of nasal mucosa, HE staining; Infiltration of the eosinophils, Sirius Red staining; Goblet cell hyperplasia, PAS staining; Collagen deposition, Masson's Trichrome staining. Scale bar $=50 \mu \mathrm{m}$ in the nasal tissues and $100 \mu \mathrm{m}$ in the lungs. Values are presented as the mean $\pm \mathrm{SD}$ ( $\mathrm{n}=6$ per group). \#\#\# $P<0.001$, significant difference of the control group. ${ }^{* *} P<0.01,{ }^{* * *} P<0.001$, significant differences of the OVA group.

Figure 3. Effect of SSD on the level of OVA-specific IgE, IgG1, and IgG2a expression in OVAinduced AR mice. The serum levels of OVA-specific IgE, $\operatorname{IgG}_{1}$, and IgG2a. Values are presented as the mean $\pm \mathrm{SD}(\mathrm{n}=6$ per group). \# $<0.05$, \#\# $<0.01$, \#\#\#P $<0.001$, significant differences of the control group. ${ }^{*} \mathrm{P}<0.05,{ }^{* *} \mathrm{P}<0.01,{ }^{* * *} \mathrm{P}<0.001$, significant differences of the OVA group.

Figure 4. Effect of SSD on Th1/Th2/Th17 cytokines and related transcription factor levels and NF-KB p65 and P-NF-B signaling pathways in OVA-induced AR mice. The levels of (A), Th1 cytokines, IL-12, and IFN- $\gamma \cdot$ Th2 cytokines, IL-5, IL-13, and TNF- $\alpha \cdot$ and Th17 cytokines, IL-17, were determined in NALF and (B) lung homogenates in OVA-induced AR mice. (C) Analysis of T-bet, GATA-3, and ROR $\gamma t$ expression levels in lung homogenates. (D) Detection of NF- $x \mathrm{~B}$ and P-NF- $x \mathrm{~B}$ in NALF and lung homogenates. Values are presented as the mean $\pm \mathrm{SD}\left(\mathrm{n}=6\right.$ per group). ${ }^{*} P<0.05$, \#\# $P<0.01$, \#\#\# $P$ $<0.001$, significant differences of the control group. ${ }^{*} P<0.05,{ }^{* *} P<0.01,{ }^{* * *} P<0.001$, significant differences of the OVA group. 
A
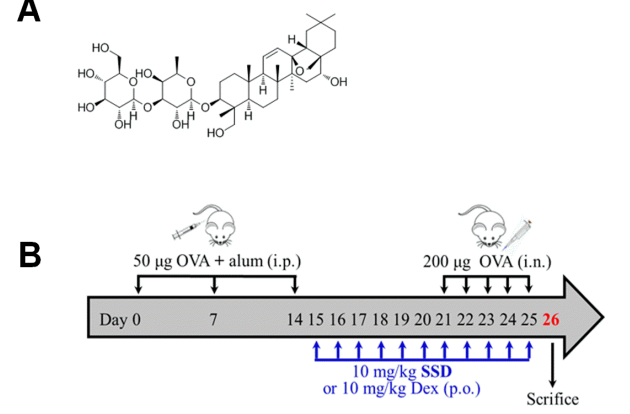

C
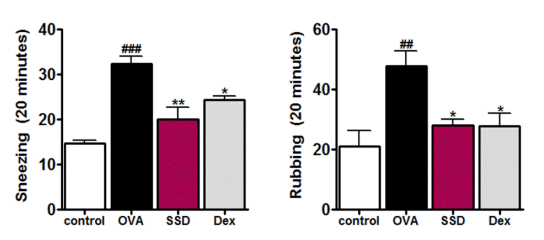

D

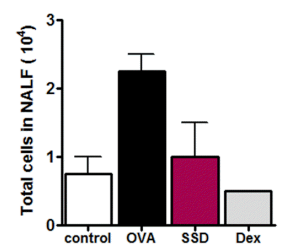

E
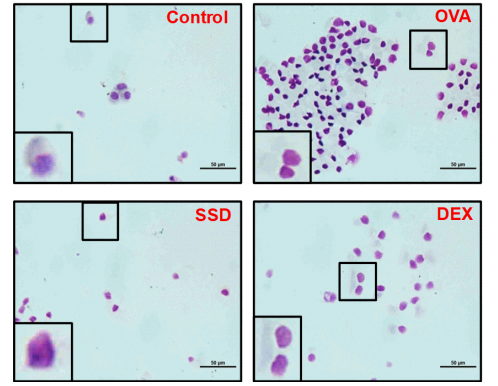

A

B
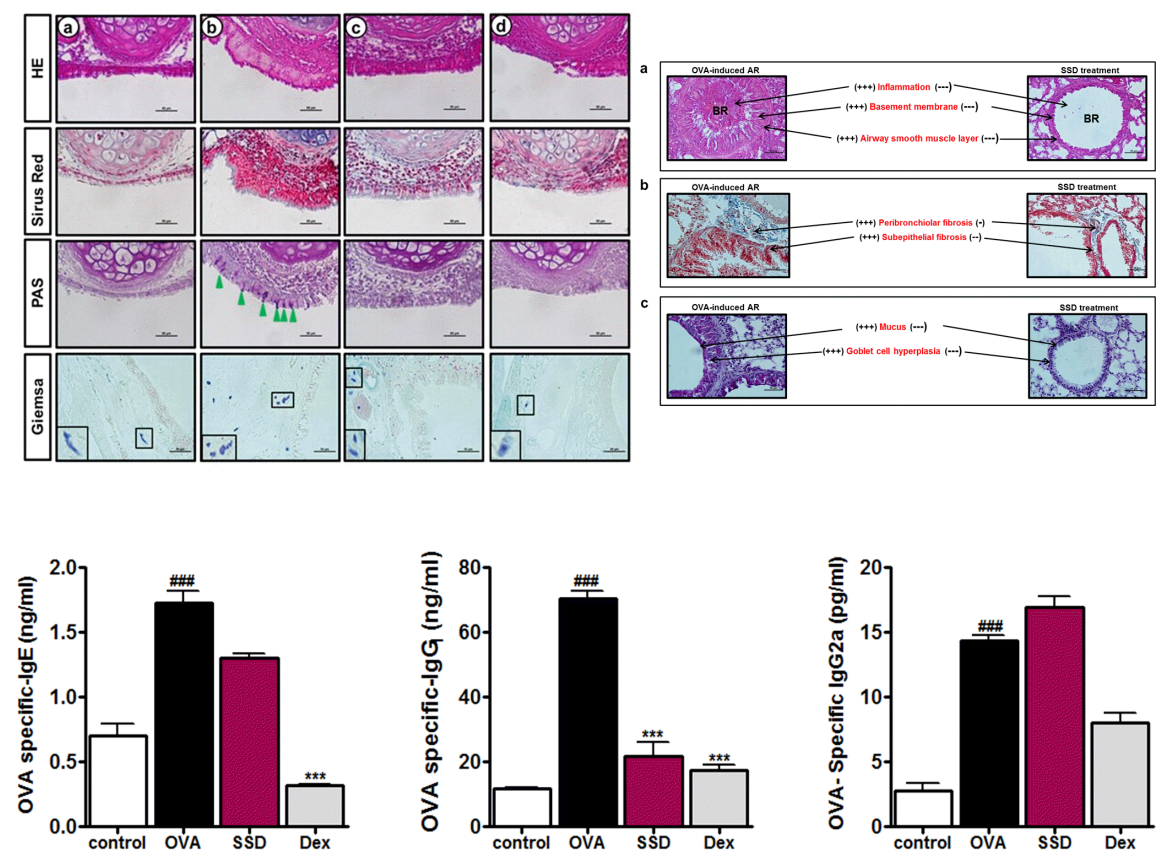


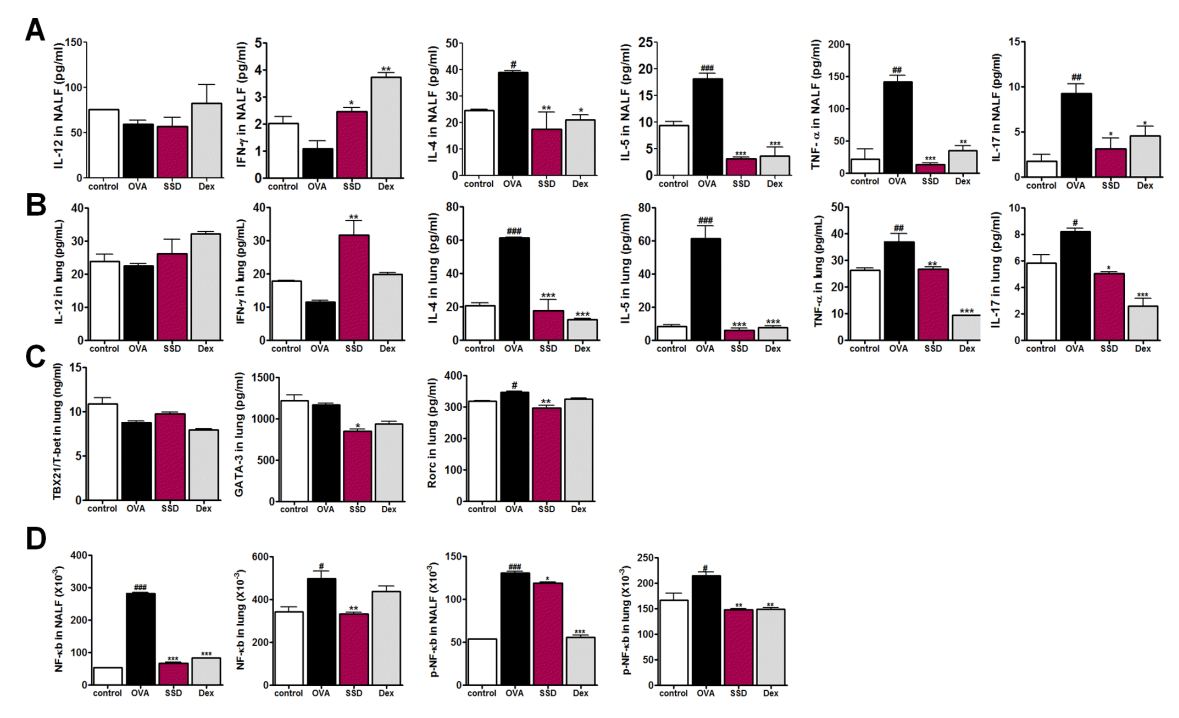

